\title{
PRODUÇÃO DE PAINÉIS COMPENSADOS COM LÂMINAS DE MADEIRA DE Sequoia sempervirens E RESINA UREIA- FORMALDEÍDO
}

\author{
Setsuo Iwakiri ${ }^{1}$, Alexsandro Bayestorff Cunha ${ }^{2}$, José Guilherme Prata ${ }^{1}$, Rafael Leite Braz ${ }^{3}$, \\ Vinicius Gomes Castro ${ }^{3}$, Sandra Kazmierczak ${ }^{3}$, Edson Pinheiro ${ }^{3}$, Heloisa Rancatti ${ }^{3}$, Felipe Luis Sanches ${ }^{3}$ \\ ${ }^{1}$ Eng. Florestal, Dr., Depto. Engenharia e Tecnologia Florestal, UFPR, Curitiba, PR, Brasil - setsuo@ufpr.br, jgprata@ufpr.br \\ ${ }^{2}$ Eng. Florestal, Dr., Depto. Engenharia Florestal, UDESC, Lajes, SC, Brasil - a2abc@cav.udesc.br \\ ${ }^{3}$ Eng. Florestal, Doutorando em Engenharia Florestal, PPGEF, UFPR, Curitiba, PR, Brasil - rafaellbraz@ yahoo.com.br, \\ vinnie.castro@gmail.com. \\ ${ }^{4}$ Eng. Florestal, Mestrando em Engenharia Florestal, PPGEF, UFPR, Curitiba, PR, Brasil - Sandra_kazi@ @otmail.com, \\ Edson@arbon.com.br, helorancatti@yahoo.com.br, engenhariaflorestal.felipe@gmail.com
}

Recebido para publicação: 24/01/2012 - Aceito para publicação: 12/12/2012

\section{Resumo}

Esta pesquisa teve por objetivo avaliar a qualidade dos painéis compensados multilaminados produzidos com lâminas de madeira de Sequoia sempervirens (D. Don) Endl. Foram produzidos em laboratório compensados com cinco lâminas de 2,0 mm de espessura, colados com resina ureia-formaldeído (UF), com duas diferentes formulações de batida de cola e gramaturas. Os painéis foram prensados com pressão específica de $10 \mathrm{kgf} / \mathrm{cm}^{2}$, temperatura de $110{ }^{\circ} \mathrm{C}$ e tempo de prensagem de 10 minutos. Foram avaliadas as propriedades de resistência da linha de cola aos esforços de cisalhamento (RLC) e flexão estática paralelo e perpendicular (MOE e MOR). De uma forma geral, as diferentes formulações da batida de cola e gramaturas não afetaram significativamente os resultados das propriedades dos painéis, o que representa um aspecto importante sob o ponto de vista econômico. Os valores médios de RLC e percentagem de falhas na madeira atendem aos requisitos mínimos da norma EN 314-2 (1993). Os resultados obtidos nesta pesquisa indicam a possibilidade de uso de lâminas de sequoia para o miolo de painéis compensados para uso interior e de forma associada com lâminas de espécies com maior resistência mecânica nas capas.

Palavras-chave: Lâminas de madeira; sequoia; compensado; ureia-formaldeído.

\begin{abstract}
Production of plywood using veneers of Sequoia sempervirens and urea-formaldehyde resin. This research was carried out to evaluate the quality of plywood made from wood veneer of Sequoia sempervirens. It was produced in laboratory plywood composed by five veneers with $2.0 \mathrm{~mm}$ thick, bonded with urea-formaldehyde resin (UF) with two different formulations of glue and amount of glue spread. The panels were pressed with specific pressure of $10 \mathrm{kgf} / \mathrm{cm}^{2}$, temperature of $110{ }^{\circ} \mathrm{C}$ and pressing time of 10 minutes. It was evaluated the properties of the glue line shear strength (RLC) and static bending (MOE and MOR) parallel and perpendicular. In general, the different adhesive formulations and amount of spread glue did not significantly affect the results of the properties of the panels, which represent an important aspect on the economic point of view. The mean values of RLC and percentage of wood failure meet minimum requirements of standard EN 314-2 (1993). The results of this research revealed the possibility to using the veneers of sequóia for the core of internal use plywood associated with greater strength species on the faces.

Keywords: Wood veneer; sequoia; plywood; urea-formaldehyde.
\end{abstract}

\section{INTRODUÇÃO}

Estudos sobre espécies de rápido crescimento provenientes de plantios florestais para produção de lâminas e compensados têm sido amplamente realizados no Brasil, com o objetivo de aumentar a quantidade de espécies alternativas ao Pinus, que é a principal matéria-prima utilizada pelas indústrias de compensados.

Além das espécies do gênero Eucalyptus, vários pesquisadores estudaram o comportamento de diversas espécies para produção de lâminas e compensados. Pinto (2011) avaliou o rendimento em 
laminação e qualidade dos painéis compensados de Criptomeria japonica (L. f.) D. Don; Bortoletto Jr. e Belini (2002) estudaram o comportamento da madeira de Schizolobium parahyba Vell. Blake para produção de compensados; e Iwakiri et al. (2011) avaliaram a qualidade de painéis compensados produzidos com lâminas de Schizolobium amazonicum Huber ex. Apesar da baixa densidade da madeira dessas espécies, os pesquisadores encontraram resultados que indicam o seu potencial para produção de lâminas e compensados.

A Sequoia sempervirens é uma espécie nativa dos Estados Unidos da América e sua madeira apresenta cerne marrom-avermelhado e alburno com tonalidade branca. A sua madeira, apesar da densidade baixa $\left(0,31 \mathrm{~g} / \mathrm{cm}^{3}\right)$, possui boa estabilidade dimensional e resistência à deterioração, além de outras características favoráveis para fabricação de painéis e produção de celulose (DIEL; FRIZZO, 2002; SLOAN; BOE, 1974). Spichinger (2004) relata que a madeira de sequoia apresenta, como características, boa usinabilidade e boa aderência para tintas e vernizes, além de não apresentar resinas, típicas de madeira de coníferas. Essas qualidades tornam a madeira de sequoia atrativa para a produção de painéis e uso na indústria moveleira.

As limitações relacionadas à baixa densidade da madeira de espécies de florestas plantadas de rápido crescimento e pequenos diâmetros das árvores podem ser minimizadas com uso de tecnologias de colagem para fabricação de produtos de madeira reconstituída, como painéis laminados de madeira. Bendsten (1978) afirma que, para se alcançar êxito no uso dos recursos florestais provenientes de florestas plantadas, os conceitos tradicionais devem ser modificados e adaptados às características da matéria-prima disponível.

Na produção de compensados, os fatores relacionados à tecnologia devem ser avaliados com muita atenção, principalmente quanto ao processo físico-químico envolvido na colagem de lâminas de madeira (MARRA, 1992). O tipo de resina empregado na colagem deve ser adequado ao ambiente de utilização do painel, podendo ser ureia-formaldeído para ambiente interno e fenol-formaldeído para ambiente externo.

A formulação da batida de cola e a gramatura são parâmetros que irão influenciar diretamente na qualidade da colagem e, ao mesmo tempo, no custo de produção do compensado (BALDWIN, 1993; SELLERS, 1993). Os autores afirmam que a densidade da madeira é um fator importante na definição desses parâmetros, tendo em vista as interações que ocorrem entre a porosidade da madeira e a absorção do adesivo na formação da ligação adesiva entre as lâminas. De acordo com Marra (1992), madeiras de baixa densidade absorvem maior quantidade de adesivo, devido à sua maior porosidade, de modo que a viscosidade do adesivo deve ser aumentada, para evitar a formação da linha de cola "faminta".

Outros fatores, como pH e extrativos presentes na madeira, são também importantes, podendo interferir na cura do adesivo durante o processo de prensagem do painel na prensa quente. Segundo Marra (1992), espécies com pH muito baixo aceleram o processo de polimerização da resina ureia-formaldeído, podendo ocasionar a pré-cura da linha de cola, reduzindo a resistência da ligação adesiva. Baldwin (1993) afirma que alguns extrativos presentes nas lâminas podem dificultar o processo de vaporização e sua migração de uma linha de cola para outra, e destas para as bordas do painel, com posterior liberação para o ambiente externo. Esse processo, sendo muito lento, terá como consequência o aumento da pressão interna de vapor, resultando no "estouro" no momento da abertura da prensa e delaminação do painel.

Tendo em vista a necessidade de ampliar a oferta de madeiras de espécies de rápido crescimento provenientes de plantios florestais, este trabalho teve como objetivo avaliar o potencial de uso da espécie Sequoia sempervirens para produção de lâminas e painéis compensados para uso interno, com diferentes formulações da batida de cola e gramaturas.

\section{MATERIAL E MÉTODOS}

Foi utilizada nesta pesquisa madeira de Sequoia sempervirens com 18 anos de idade, proveniente de plantio localizado na Estação Experimental da EPAGRI de São Joaquim, estado de Santa Catarina. Para a laminação, foram utilizadas 6 toras com comprimento de $0,60 \mathrm{~m}$, obtidas a partir de três árvores coletadas aleatoriamente no plantio. As toras foram transportadas para o Laboratório de Painéis de Madeira da UFPR, onde foram descascadas e submetidas ao processo de aquecimento à temperatura em torno de $60{ }^{\circ} \mathrm{C}$, por período de 12 horas. As lâminas foram obtidas num torno piloto com espessura de $2,5 \mathrm{~mm}$ e foram seccionadas numa guilhotina pneumática com as dimensões de 600 x $600 \mathrm{~mm}$. A massa 
específica aparente e massa específica básica da madeira foram determinadas em amostras retiradas de lâminas com dimensões de 20 x $300 \mathrm{~mm}$.

Para a produção de painéis compensados, as lâminas foram secas em estufa ao teor de umidade médio de $6 \%$ e esquadrejadas em dimensões finais de 500 x $500 \mathrm{~mm}$. Os painéis foram produzidos com cinco lâminas utilizando-se resina ureia-formaldeído, com duas formulações de batida de cola e duas gramaturas, conforme apresentado na tabela 1 . Os painéis foram prensados à temperatura de $110^{\circ} \mathrm{C}$, pressão específica de $10 \mathrm{kgf} / \mathrm{cm}^{2}$ e tempo de prensagem de 8 minutos. Foram produzidos dois painéis por tratamento.

Após a prensagem, os painéis foram esquadrejados e acondicionados na câmara climática à temperatura de $20 \pm 3{ }^{\circ} \mathrm{C}$ e umidade relativa de $65 \pm 5 \%$, até sua estabilização. Para cada tratamento, foram retirados 5 corpos de prova para ensaios de flexão estática com a direção das fibras das lâminas da capa paralela ao comprimento do corpo de prova e 5 na direção perpendicular. Para ensaios de resistência da linha de cola aos esforços de cisalhamento, foram retirados 10 corpos de prova para teste seco e 10 para teste úmido. Os ensaios foram conduzidos de acordo com a Norma Europeia EN 310 e EN 314, respectivamente. Na figura 1 encontra-se ilustrado o esquema de retirada de corpos de prova nos dois painéis de cada tratamento.

Tabela 1. Delineamento experimental.

Table 1. Experimental chart.

\begin{tabular}{llcc}
\hline Tratamento & Formulação & TS (\%) & Gramatura $\left(\mathbf{g} / \mathbf{m}^{2}\right)$ \\
\hline T1 & A: $R=100 ; E=20 ; C=1,5 ; \mathrm{Ag}=20$ & 49,95 & 280 \\
T2 & $A: R=100 ; E=20 ; C=1,5 ; \mathrm{Ag}=20$ & 49,95 & 320 \\
T3 & B: $\mathrm{R}=100 ; \mathrm{E}=40 ; \mathrm{C}=1,7 ; \mathrm{Ag}=40$ & 35,80 & 280 \\
T4 & B: $\mathrm{R}=100 ; \mathrm{E}=40 ; \mathrm{C}=1,7 ; \mathrm{Ag}=40$ & 35,80 & 320 \\
\hline
\end{tabular}

UF: ureia-formaldeído; R: resina; E: extensor - farinha de trigo; $\mathrm{C}$ : catalisador; Ag: água.

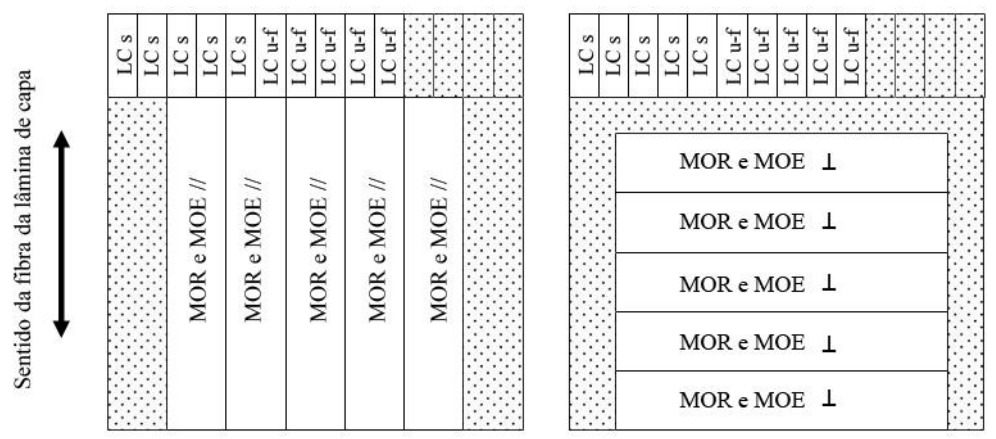

Figura 1. Representação do esquema de retirada dos corpos de prova.

Figure 1. Schematic drawing to samples cutting.

O delineamento estatístico utilizado foi o inteiramente casualizado e os resultados dos ensaios foram analisados através da análise de variância e teste de Tukey ao nível de probabilidade de $95 \%$.

\section{RESULTADOS E DICUSSÃO}

\section{Lâminas de madeira}

Os valores médios de massa específica aparente e massa específica básica da madeira de sequoia determinados a partir de lâminas foram, respectivamente, de $0,340 \mathrm{~g} / \mathrm{cm}^{3}$ e $0,290 \mathrm{~g} / \mathrm{cm}^{3}$. Esses valores são considerados baixos quando comparados aos valores referenciados na literatura para espécies provenientes de plantios florestais. Trianoski (2010) obteve os seguintes valores de massa específica básica para as espécies estudadas: Pinus taeda L. $\left(0,496 \mathrm{~g} / \mathrm{cm}^{3}\right)$, Acrocarpus fraxinifolius Wight e Arn Mundanu $\left(0,458 \mathrm{~g} / \mathrm{cm}^{3}\right)$, Melia azedarach L. $\left(0,488 \mathrm{~g} / \mathrm{cm}^{3}\right)$, Gravillea robusta Cunn $\left(0,494 \mathrm{~g} / \mathrm{cm}^{3}\right)$, Schizolobium parahyba Vell. Blake $\left(0,264 \mathrm{~g} / \mathrm{cm}^{3}\right)$ e Toona ciliata M. Roem $\left(0,373 \mathrm{~g} / \mathrm{cm}^{3}\right)$. Bortoletto Jr. 
(2003) obteve, para 11 espécies do gênero Eucalyptus provenientes de plantios florestais, valores de massa específica básica variando de $0,560 \mathrm{~g} / \mathrm{cm}^{3}$ a $0,770 \mathrm{~g} / \mathrm{cm}^{3}$.

O rendimento total médio obtido para as 6 toras laminadas foi de $41,21 \%$, com base no volume das toras sem casca, e o rendimento efetivo médio de $55,67 \%$, com base no volume das toras após o arredondamento. Essa diferença entre os rendimentos pode ser atribuída ao alto grau de conicidade das toras. As avaliações da qualidade das lâminas demonstraram a presença de nós em $100 \%$ das lâminas, sendo um indicativo de que as árvores desses plantios experimentais não passaram por procedimentos de desrama. Foram observadas também presença de lâminas com rugosidades e fendas superficiais.

Os resultados de rendimento obtidos nesta pesquisa são inferiores quando comparados com os valores apresentadas na literatura para espécies de pínus e eucaliptos provenientes de plantios florestais. Almeida et al. (2004) obtiveram, para clones I e II de Eucalyptus grandis Hill ex. Maiden $x$ Eucalyptus urophylla S. T. Blake, rendimento médio de $51,74 \%$ e 56,81\%, respectivamente. Pio (1996) encontrou, para espécies de Eucalyptus scabra Dum-Cours e Eucalyptus robusta Smith, rendimento médio de $36,47 \%$ e $44,00 \%$, respectivamente. O autor cita ainda como fontes de referência os rendimentos médios para as seguintes espécies de pínus: Pinus elliottii Engelm (42,40\%), Pinus taeda L. (55,50\%) e Pinus strobus L. Small (54,40\%). Interamnense (1998) obteve, para espécies de Eucalyptus viminalis Labill., Eucalyptus cloeziana F. Muell e Eucalyptus maculata Hook, valores médios de rendimento de 50,00\%, $50,43 \%$ e $44,86 \%$, respectivamente.

\section{Resistência da linha de cola às tensões de cisalhamento}

Na tabela 2 estão apresentados os valores médios de resistência da linha de cola às tensões de cisalhamento e percentagens de falhas na madeira.

Tabela 2. Resultados dos ensaios de cisalhamento da linha de cola.

Table 2. Results of glue line shear test.

\begin{tabular}{lcccccc}
\hline \multirow{3}{*}{ Tratamento } & \multicolumn{3}{c}{ Teste seco } & \multicolumn{3}{c}{ Teste úmido } \\
\cline { 2 - 7 } & $\begin{array}{c}\text { Média } \\
(\mathbf{M P a})\end{array}$ & $\begin{array}{c}\text { CV } \\
\mathbf{( \% )}\end{array}$ & $\begin{array}{c}\text { FM } \\
\mathbf{( \% )}\end{array}$ & $\begin{array}{c}\text { Média } \\
(\mathbf{M P a})\end{array}$ & $\begin{array}{c}\text { CV } \\
\mathbf{( \% )}\end{array}$ & $\begin{array}{c}\text { FM } \\
(\boldsymbol{\%})\end{array}$ \\
\hline T1 & $1,03 \mathrm{~b}$ & 23,04 & 65 & $0,62 \mathrm{c}$ & 37,10 & 20 \\
$\mathrm{~T} 2$ & $1,36 \mathrm{a}$ & 20,23 & 81 & $1,25 \mathrm{a}$ & 10,28 & 68,5 \\
T3 & $1,00 \mathrm{~b}$ & 21,14 & 84 & $0,79 \mathrm{bc}$ & 31,13 & 49,5 \\
T4 & $1,15 \mathrm{ab}$ & 17,93 & 75 & $1,02 \mathrm{ab}$ & 19,93 & 52 \\
\hline
\end{tabular}

Médias seguidas pela mesma letra não diferem estatisticamente entre si a 95\% de probabilidade. CV: coeficiente de variação; FM: falha na madeira.

Os resultados indicam que não houve influência significativa da formulação da batida de cola sobre a resistência da linha de cola dos painéis (T1 x T3 e T2 x T4), tanto para o teste seco quanto para o teste úmido. Portanto, o aumento no teor de sólidos da batida de cola não resultou em incremento nos valores dessa propriedade, o que significa vantagens em termos econômicos, devido à redução na proporção de resina na mistura.

Com relação ao efeito da gramatura, tanto para o teste seco quanto para o teste úmido, nos painéis produzidos com a formulação $\mathrm{A}$ (T1, T2) foram constatados aumentos estatisticamente significativos da resistência da linha de cola para incrementos em gramatura de $280 \mathrm{~g} / \mathrm{m}^{2}$ para $320 \mathrm{~g} / \mathrm{m}^{2}$. Já para os painéis produzidos com a formulação B (T3, T4), não foram constatadas diferenças estatisticamente significativas entre as duas gramaturas de cola, embora, em termos de médias absolutas, o aumento na gramatura de $280 \mathrm{~g} / \mathrm{m}^{2}$ para $320 \mathrm{~g} / \mathrm{m}^{2}$ tenha resultado em maiores valores médios de resistência da linha de cola.

Na comparação com os valores referenciais apresentados na literatura, os resultados de RLC teste seco obtidos nesta pesquisa foram superiores aos valores médios obtidos por Iwakiri et al. (2011), na faixa de 0,72 a 0,94 MPa, para painéis compensados de Schizolobium amazonicum Huber ex. (paricá) produzidos com resina ureia-formaldeído. Numa outra pesquisa, Iwakiri et al. (2002) encontraram, para painéis compensados ureicos de Pinus taeda L., valores de RLC na faixa de 1,06 a 1,57 MPa. 
Com exceção do tratamento T1 (teste úmido), os valores médios de resistência da linha de cola obtidos para os compensados de sequoia atendem aos requisitos mínimos da norma EUROPEAN COMMITTEE FOR STANDARDIZATION (EN 314-2: 1993) para painéis de uso interior, cujos valores são de 0,6 a $1,0 \mathrm{MPa}$, associados à percentagem de falhas na madeira superior a $40 \%$.

\section{Flexão estática paralela}

Na tabela 3 estão apresentados os valores médios de massa específica, módulo de elasticidade e módulo de ruptura em flexão estática paralela dos painéis.

Tabela 3. Resultados de massa específica e flexão estática paralela.

Table 3. Results of board density and parallel static bending.

\begin{tabular}{lcccccc}
\multirow{2}{*}{ Tratamento } & \multicolumn{2}{c}{$\mathbf{M E}\left(\mathbf{g} / \mathbf{c m}^{3}\right)$} & \multicolumn{2}{c}{ MOE (MPa) } & \multicolumn{2}{c}{ MOR (MPa) } \\
\cline { 2 - 7 } & Média & $\mathbf{C V ~}(\boldsymbol{\%})$ & Média & $\mathbf{C V}(\boldsymbol{\%})$ & Média & CV $(\boldsymbol{\%})$ \\
\hline T1 & 0,413 & 4,15 & $2.718 \mathrm{~b}$ & 11,59 & $23 \mathrm{a}$ & 20,90 \\
T2 & 0,427 & 4,08 & $3.503 \mathrm{a}$ & 12,37 & $26 \mathrm{a}$ & 29,29 \\
T3 & 0,407 & 2,87 & $3.241 \mathrm{ab}$ & 11,33 & $23 \mathrm{a}$ & 26,54 \\
T4 & 0,423 & 1,69 & $2.834 \mathrm{~b}$ & 11,06 & $24 \mathrm{a}$ & 3,78 \\
\hline
\end{tabular}

Médias seguidas pela mesma letra não diferem estatisticamente entre si a 95\% de probabilidade. ME: massa específica; MOE: módulo de elasticidade; MOR: módulo de ruptura; CV: coeficiente de variação.

Os valores médios da massa específica dos painéis variaram de 0,407 a $0,427 \mathrm{~g} / \mathrm{cm}^{3}$, sendo esses valores inferiores em comparação aos painéis compensados comerciais de Pinus taeda L., com média em torno de $0,53 \mathrm{~g} / \mathrm{cm}^{3}$ (ASSOCIAÇÃO BRASILEIRA DA INDÚSTRIA DA MADEIRA PROCESSADA MECANICAMENTE (ABIMCI), 2002).

As diferentes formulações da batida de cola influenciaram de forma positiva apenas os painéis produzidos com gramatura de $320 \mathrm{~g} / \mathrm{m}^{2}$ (T2 x T4), em que a formulação A, com maior proporção de resina UF, resultou em maior MOE paralelo. Já para gramatura de $280 \mathrm{~g} / \mathrm{m}^{2}$ não foi constatada diferença estatisticamente significativa entre os painéis produzidos com diferentes formulações (T1 x T3). Para gramaturas diferentes, foram constatadas diferenças significativas apenas para os painéis produzidos com formulação A (T1 x T2), em que o aumento na gramatura de 280 para $320 \mathrm{~g} / \mathrm{m}^{2}$ resultaram em painéis com maior MOE paralelo.

Para o MOR paralelo não foram constatadas diferenças estatisticamente significativas entre os tratamentos. As diferentes formulações da batida de cola e gramaturas não influenciaram de forma significativa os resultados dessa propriedade.

Os resultados de MOE e MOR paralelo obtidos para os compensados de sequoia foram inferiores em comparação aos valores obtidos por Iwakiri et al. (2002) para painéis de Pinus taeda L., cujos valores médios foram, respectivamente, de 4.330 MPa e 32,0 MPa. Bortoletto Jr; Belini (2002) obtiveram, para painéis compensados de Schizolobium parahyba (guapuruvu), valores de MOE e MOR paralelo de 6.425 MPa e 51,7 MPa, respectivamente. As diferenças verificadas com relação aos painéis de sequoia podem ser atribuídas à maior massa específica dos painéis compensados de Pinus taeda, de $0,500 \mathrm{~g} / \mathrm{cm}^{3}$, e de Schizolobium parahyba, de $0,462 \mathrm{~g} / \mathrm{cm}^{3}$, que contribuíram para a obtenção de maiores valores de MOE e MOR paralelo em flexão estática.

Com relação aos painéis comerciais de Pinus taeda L., os dados de MOE e MOR paralelo apresentados no catálogo técnico da ABIMCI (2002) são, respectivamente, de $6.890 \mathrm{MPa}$ e $38,1 \mathrm{MPa}$, para massa específica dos painéis de $0,530 \mathrm{~g} / \mathrm{cm}^{3}$. Portanto, a densidade das lâminas de madeira utilizadas na manufatura de compensados tem uma forte influência nas propriedades de MOE e MOR em flexão estática.

\section{Flexão estática perpendicular}

$\mathrm{Na}$ tabela 4 estão apresentados os valores médios de massa específica, módulo de elasticidade e módulo de ruptura em flexão estática perpendicular dos painéis. 
Tabela 4. Resultados de massa específica e flexão estática perpendicular.

Table 4. Results of board density and perpendicular static bending.

\begin{tabular}{lcccccc}
\hline \multirow{2}{*}{ Tratamento } & \multicolumn{2}{c}{ ME $\left(\mathbf{g} / \mathbf{c m}^{\mathbf{3}}\right)$} & \multicolumn{2}{c}{ MOE $(\mathbf{M P a})$} & \multicolumn{2}{c}{ MOR (MPa) } \\
\cline { 2 - 7 } & Média & $\mathbf{C V ~ ( \% )}$ & Média & $\mathbf{C V ~ ( \% )}$ & Média & CV (\%) \\
\hline T1 & 0,425 & 3,41 & $1.084 \mathrm{a}$ & 22,06 & $13 \mathrm{a}$ & 27,03 \\
T2 & 0,432 & 3,24 & $1.305 \mathrm{a}$ & 19,41 & $14 \mathrm{a}$ & 23,21 \\
T3 & 0,425 & 4,98 & $1.051 \mathrm{a}$ & 28,35 & $13 \mathrm{a}$ & 2,02 \\
T4 & 0,415 & 3,67 & $1.080 \mathrm{a}$ & 11,14 & $8 \mathrm{a}$ & 37,20 \\
\hline
\end{tabular}

Médias seguidas pela mesma letra não diferem estatisticamente entre si a 95\% de probabilidade. ME: massa específica; MOE: módulo de elasticidade; MOR: módulo de ruptura; CV: coeficiente de variação.

Para as amostras de painéis utilizadas para ensaios de flexão estática perpendicular, os valores médios de massa específica variaram na faixa de 0,415 a $0,432 \mathrm{~g} / \mathrm{cm}^{3}$.

Tanto para o MOE quanto para o MOR perpendicular, as diferentes formulações da batida de cola e gramaturas de 280 e $320 \mathrm{~g} / \mathrm{m}^{2}$ não influenciaram de forma estatisticamente significativa nos valores médios dessas propriedades. Esses resultados são importantes sob o ponto de vista econômico, devido à possibilidade de redução no consumo de cola.

O catálogo técnico da ABIMCI (2002) apresenta valores médios de MOE e MOR perpendicular, respectivamente, de $2.839 \mathrm{MPa}$ e 25,3 $\mathrm{MPa}$, para painéis comerciais de Pinus taeda L. com massa específica de $0,53 \mathrm{~g} / \mathrm{cm}^{3}$. Os resultados obtidos para painéis de sequoia foram inferiores a esses valores, tendo em vista sua menor massa específica.

\section{CONCLUSÕES}

Com base nos resultados obtidos, as seguintes conclusões podem ser apresentadas:

- O rendimento médio em laminação foi de $41,21 \%$, e a massa específica aparente média, de $0,340 \mathrm{~g} / \mathrm{cm}^{3}$.

- A formulação da batida de cola com maior proporção de resina UF influenciou de forma positiva apenas no MOE paralelo dos painéis produzidos com gramatura de $320 \mathrm{~g} / \mathrm{m}^{2}$. Esses resultados são importantes sob o ponto de vista econômico, devido à possibilidade de redução no consumo de cola.

- A gramatura influenciou de forma positiva apenas no MOE paralelo dos painéis produzidos com maior teor de sólidos da batida de cola (formulação A).

- A resistência da linha de cola dos painéis atende aos requisitos da Norma Europeia EN 314-2 (1993).

- Os painéis compensados de sequoia apresentaram baixa massa específica, o que contribuiu para redução nos resultados de MOE e MOR em flexão estática.

- Os resultados indicam a possibilidade de uso de lâminas de sequoia para o miolo de painéis compensados para uso interno e de forma associada com lâminas de espécies com maior resistência mecânica nas capas.

\section{AGRADECIMENTOS}

Os autores expressam seus agradecimentos à EPAGRI - Estação Experimental de São Joaquim SC, pela doação da madeira de Sequoia sempervirens utilizada nesta pesquisa.

\section{REFERÊNCIAS}

ALMEIDA, R. R.; BORTOLETTO JR., G.; JANKOWSKI, I. P. Produção de lâminas a partir da madeira de clones do híbrido Eucalyptus grandis $x$ Eucalyptus urophylla. Scientia Forestalis, Piracicaba, v. 65, p. 49 - 58, 2004.

ASSOCIAÇÃO BRASILEIRA DA INDÚSTRIA DA MADEIRA PROCESSADA MECANICAMENTE (ABIMCI). Catálogo técnico de compensados de pínus. Curitiba, 2002. 20 p.

BALDWIN, R. F. Plywood and veneer-based products: manufacturing practices. San Francisco: Miller Freeman, 1993. 388 p. 
BENDSTEN, B. A. Properties of wood from improved and intensively managed tress. In: Proceedings of the economics and financial management and timber production technical commitee of the forest products research society. Atlanta, 1978. 78 p.

BORTOLETTO JR., G.; BELINI, U. L. Produção de lâminas e manufatura de compensados a partir da madeira de guapuruvu (Schizolobium parahyba Blake) proveniente de um plantio misto de espécies nativas. Cerne, Lavras, v. 8, n. 2, p. 1 - 16, 2002.

BORTOLETTO JR., G. Produção de compensados com 11 espécies do gênero Eucalyptus, avaliação das suas propriedades físico-mecânicas e indicações para utilização. Scientia Forestalis, Piracicaba, v. 63, p. $65-78,2003$.

DIEL, J.; FIZZO, S. Estudos de caracterização da Sequoia sempervirens para produção de celulose Kraft. In: $35^{\circ}$ CONGRESSO E EXPOSIÇAO ANUAL DE CELULOSE E PAPEL. 2002, São Paulo. Anais... São Paulo, 2002, p. 55 - 74.

EUROPEAN COMMITTEE FOR STANDARDIZATION (CEN). EN 310: 1993: Plywood Determination of modulus of elasticity and modulus of rupture in static bending. 1993.

EUROPEAN COMMITTEE FOR STANDARDIZATION (CEN). EN 314-2: 1993: Plywood Determination of shear bonding strength. 1993.

INTERAMNENSE, M. T. Utilização das madeiras de Eucalyptus cloeziana (F. Muell), Eucalyptus maculata (Hook) e Eucalyptus punctata DC var. punctata para produção de painéis compensados. 81 f. Dissertação (Mestrado em Tecnologia de Produtos Florestais) - Universidade Federal do Paraná, Curitiba, 1998.

IWAKIRI, S.; SILVA, J. C.; SILVA, J. R. M.; AlVES, C. R.; PUEHRINGER, C. A. Produção de compensados de Pinus taeda L. e Pinus oocarpa Schiede com diferentes formulações de adesivo ureiaformaldeído. Árvore, Viçosa, v. 26, n. 3, p. 371 - 375, 2002.

IWAKIRI, S.; VARGAS, C. A.; PARCHEN, C. F. A.; WEBER, C.; BATISTA, C. C.; GARBE, E. A.; CIT, E. J.; PRATA, J. G. Avaliação da qualidade de painéis compensados produzidos com lâminas de madeira de Schizolobium amazonicum. Floresta, Curitiba, v. 41, n. 3, p. 451 - 458, 2011.

MARRA, A. A. 1992. Technology of wood bonding: principles in practice. New York: Van Nostrand Reinhold. 1992. 453 p.

PINTO, J. A. Análise da viabilidade do uso da madeira de Cryptomeria japônica para laminação e produção de painéis compensados. 85 f. Dissertação (Mestrado em Engenharia Florestal) Universidade Federal do Paraná, Curitiba, 2011.

PIO, N. S. Avaliação da madeira de Eucalyptus scabra (Dum-Cours) e Eucalyptus robusta (Smith) na produção de painéis compensados. $101 \mathrm{f}$. Dissertação (Mestrado em Tecnologia de Produtos Florestais) - Universidade Federal do Paraná, Curitiba, 1996.

SELLERS, T. Plywood and adhesive technology. New York: Marcel Dekker, 1993. 661 p.

SLOAN J.; BOE, K. Sequoia sempervirens (Lamb. Ex D. Don) Endl. In: Seeds of Woody plants in the United States. Schopmeyer, USA, 1974.

SPICHINGER, O. A. Aprovechamiento en el aserrado de sequoia (Sequoia sempervirens (D. DON) Endl.) y classificación de la madeira obtenida. 61 f. Dissertação (Mestrado em Ingeniería de La Madeira) - Universidad de Chile, Santiago, 2004.

TRIANOSKI, R. Avaliação do potencial de espécies florestais alternativas de rápido crescimento para produção de painéis de madeira aglomerada. 260 f. Dissertação (Mestrado em Engenharia Florestal) - Universidade Federal do Paraná, Curitiba, 2010. 
